\title{
Synthesis of Liquid Antioxidant DTBNP Catalyzed by Composite Super Acid
}

\author{
Jinting Yu and Zheng $\mathrm{Li}^{*}$
}

College of General Education, Tianjin Normal University, Tianjin 300387, P.R. China

\begin{abstract}
Di-tert-butyl-4-nonylphenol(DTBNP) was synthesized from 4-n-nonyl phenol and isobutylene, etc. using self-made composite super acid as catalyst. The appropriate technology conditions are as follows: reaction temperature is $60-80^{\circ} \mathrm{C}$, reaction time is $4 \mathrm{~h}, n(4-\mathrm{n}$-nonyl phenol) $: n$ (Isobutylene) $=1: 2$ and the amount of catalysts is $0.019 \%$ of the quantity of 4-n-nonyl phenol reactant, the yield can reach to $98 \%$.
\end{abstract}

Keywords: Liquid antioxidant DTBNP, 4-n-nonyl phenol, isobutylene, composite super acid, catalyst.

2, 6-Di-tert-butyl-4-nonylphenol (DTBNP) is a new kind of liquid hindered phenolic antioxidants. It does not have the defects shared by the traditional solid antioxidants (BHT and T501) such as low dispersibility in protective solute, yellowing and decoloring, and short time of antioxidation [1, 2], because DTBNP has a higher molecular weight than the traditional phenolic antioxidants. It has low volatility and could keep liquid state at room temperature. Therefore much attention has been focused on the research of liquid hindered phenolic antioxidants $[3,4]$.

In this research, 4-n-nonyl phenol, isobutylene and selfmade composite super acid was used to catalyze the alkylation reaction in order to synthesize DTBNP. As a parent substance of liquid hindered phenolic antioxidants, DTBNP combined with other functional additives can be made into various liquid anti-oxidizers.

\section{EXPERIMENTAL SECTION}

\subsection{Reagents and Equipments}

Reagents: 4-n-Nonyl phenol,(industrial product of 99\% purity) was purchased from Jilin chemical industry Co., LTD. Isobutylene,(industrial product of $99 \%$ purity) was purchased from Yanshan petrochemical Co., LTD. Triethanolamine (industrial product of $85 \%$ purity) was purchased from Shenyang chemical plant, Alkylation catalyst (selfmade).

Equipments: Elementar vario El Element Analyzer; Nicolet 170SX fourier transform infrared spectro-meter. Varian Mercury Vx300 Nuclear Magnetic Reso-nance Apparatus, a 4-mouth flask with agitator and thermometer, heating cover, the freeze-pillar, condenser tube, vacuum pump system, round bottom flask, isobutylene induction pipe, isobutylene storage tank.

*Address correspondence to this author at the College of General Education, Tianjin Normal University, Tianjin 300387, P.R. China; Tel: +86 22 23766375; E-mail: tjnulz@126.com

\subsection{Reaction Equation}

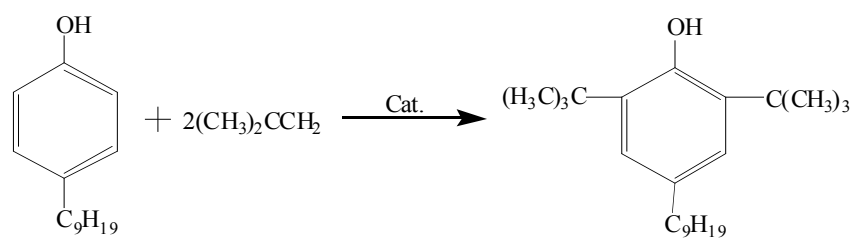

\subsection{Synthetic Methods}

\subsubsection{Choice and Preparation of the Catalyst}

It has been known that the butylation of alkyl phenol needs the usage of catalyst. The traditional catalysts include sulfate, phosphate $[5,6]$, cation exchange resin [7], $\mathrm{SO}_{4}{ }^{2-}$ $/ \mathrm{ZrO}_{2}$ supported on HMS [8] and heteropolyacid supported on $\mathrm{ZrO}_{2}$ [9]. However these catalysts more or less have the shortcomings of large dosage, strong corrosion, complex post-treatment technology, environmental pollution, deep colored products and poor reproducibility. As a result, the key issue of synthesizing 2,6-di-tert-butyl-4-nonylphenol is the choice of catalyst.

After a series of screening and research, we found that perfluoroalkyl sulfonic acid (it is mainly the trifluoromethane sulfonic acid) or Perfluoroalkyl sulfonate (it is mainly transition metal salt) is a perfect Bronsted super acid and Lewis super acid. As early as 1977 G. A. Olah pointed that perfluoroalkyl sulfonic acid or perfluoroalkyl sulfonate could replace sulphuric acid and Lewis acid to catalyze many chemical reactions $[10,11]$ and the acidity of some super acid $[12,13]$ is $10^{15}-10^{18}$ times of $100 \%$ sulphuric acid. Therefore the first choice of the catalyst of this research is the typical super acid of perfluoroalkyl sulfonic acid [14] which is equivalent to the connection of sulfonic group and fluorocarbon chain. Because the perfluoroalkyl group has a strong electrophilic effect, the degree of acidity of perfluoroalkyl group is higher than $98 \%$ sulphuric acid (see Table 1). 
Table 1. Hammett Acidity of Perfluoroalkyl Sulfonic Acid

\begin{tabular}{|c|c|}
\hline Acid & Ho(22 $\left.{ }^{\circ} \mathbf{C}\right)$ \\
\hline \hline $\mathrm{H}_{2} \mathrm{SO}_{4}$ & -12 \\
\hline $\mathrm{CF}_{3} \mathrm{SO}_{3} \mathrm{H}$ & -14.1 \\
\hline $\mathrm{C}_{2} \mathrm{~F}_{5} \mathrm{SO}_{3} \mathrm{H}$ & -14.0 \\
\hline $\mathrm{C}_{5} \mathrm{~F}_{11} \mathrm{SO}_{3} \mathrm{H}$ & -13.2 \\
\hline $\mathrm{C}_{8} \mathrm{~F}_{17} \mathrm{SO}_{3} \mathrm{H}$ & -12.3 \\
\hline
\end{tabular}

After a series of experiments we found that perfluoroalkyl sulfonic acid (it is mainly trifluoromethane sulfonic acid) had the disadvantage of strong water absorption during the process of reaction and transfer which usually results in failure of the alkylation of Friedel-Crafts. On the foundation of literature review of the previous research $[8,10,11,12,15]$, after many screening and combination tests of various kinds of perfluoroalkyl sulfonic acid and water-absorbent, a composite super acid catalyst was compounded which would be used to synthesize 2,6-ditert-butyl-4-nonylphenol. The proportion of the catalyst composite super acid is that $m\left(\mathrm{C}_{5} \mathrm{~F}_{11} \mathrm{SO}_{3} \mathrm{H}\right): m\left(\mathrm{CF}_{3} \mathrm{SO}_{3} \mathrm{H}\right)$ : $m\left(\left(\mathrm{CF}_{3} \mathrm{SO}_{2}\right)_{2} \mathrm{O}\right)=0.8: 0.1: 0.1$. This self-made catalyst has the advantage of high selectivity and catalytic activity. It has stronger acidity than inorganic acids and has the ability to promote homogeneously catalyzed reaction. It can also reduce complex post-treatment procedure and the discharge of three wastes.

\subsubsection{The Synthesis of 2,6-Di-Tert-Butyl-4-Nonylphenol}

$464 \mathrm{~mL}$ (about 2mol) 4-n-nonyl phenol was put into 1000 $\mathrm{mL}$ four-mouth flask with the stirrer, thermometer, condenser, and stirred and heated to the reaction temperature of $70{ }^{\circ} \mathrm{C}$. Then $0.05 \mathrm{~mL}$ catalyst was put into the flask. After 4-n-nonyl phenol and catalyst were fully mixed, isobutylene was injected into the flask. Because above reaction is an exothermic reaction, water bath should be used to cool down the flask in order to keep the reaction temperature at $70{ }^{\circ} \mathrm{C}$. After all the theoretical quantity (about 4mol) of isobutylene was injected into flask and gas flow meter floater kept stable, the reaction stopped (the reaction time is about $4 \mathrm{~h}$ ). The upper threshold of the flask was opened to release the pressure in it. Neutralizing agent(triethanolamine) was added to neutralize the reaction product until its $\mathrm{pH}$ is 7 . Then reaction product was cooled to room temperature. The next step is to refit the equipment and to fix the Kirschner distillation head, condenser, condenser column, vacuum pump and receiving flask to the above mentioned reaction flask. Finally the vacuum pump and heating jacket were started to distill the product and the distillate fraction of boiling point at $189-193{ }^{\circ} \mathrm{C}$ and $6 \mathrm{~mm} \mathrm{Hg}$ was collected. The product of distillation weighs $650 \mathrm{~g}$ and the yield of product is $98 \%$.

\section{RESULT AND DISCUSSION}

\subsection{Spectrum Analysis}

\subsubsection{IR Spectrum Analysis}

Fig. (1) shows there is a broad and strong stretching vibration absorption peak of multi-molecule associated matter of 4-n-nonyl phenol in the place of $3332.85 \mathrm{~cm}^{-1}$.
From Fig. (2) the IR spectrum of distillation product it can be seen there is a free stretching vibration absorption peak in the place of $3649.65 \mathrm{~cm}^{-1}$ which shows there is a bulky ortho position substituted alkyl (tert-butyl) in product molecules. As a result the steric hindrance makes it difficult for product molecules to associate with each other. In addition, the characteristic bands ${ }^{[15]}$ of three substituted alkyl groups in product molecules exist at $1156.67 \mathrm{~cm}^{-1}, 767.97 \mathrm{~cm}^{-1}$, $1236.95 \mathrm{~cm}^{-1}, 1438.84 \mathrm{~cm}^{-1}$. All the characterization proves that the main distillate fraction is the target product of 2,6-ditert-butyl-4- nonylphenol.

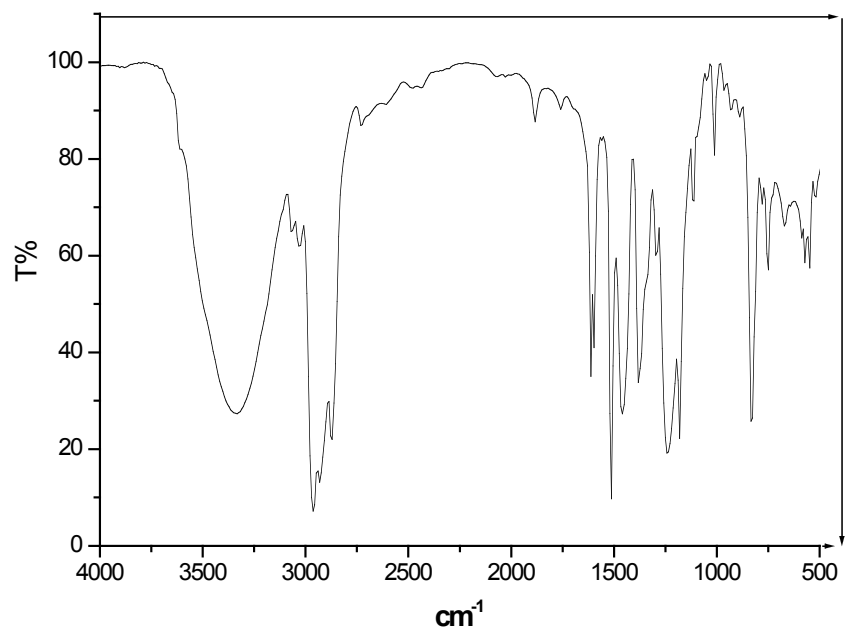

Fig. (1). IR spectrum of 4-n-nonyl phenol.

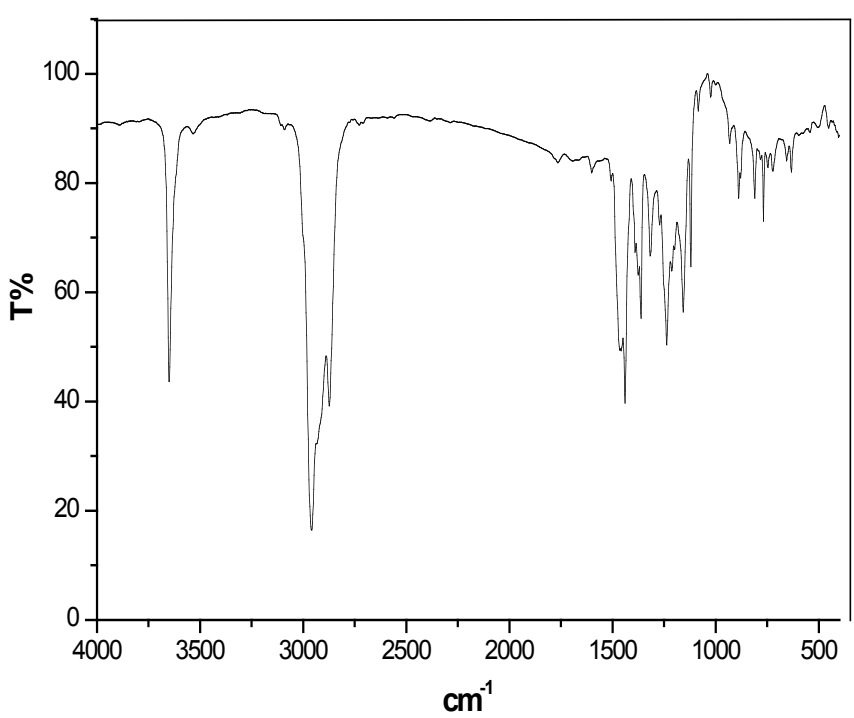

Fig. (2). IR spectrum of 2, 6-di-tert-butyl-4-nonylphenol.

\subsubsection{HNMR Spectrum Analysis}

HNMR of the synthesis product is as follows: HNMR(CDC13), $d: 0.88\left(-\mathrm{CH}_{3}\right)_{7}, \quad d: 1.58,1.43,1.23 .1 .13(-$ $\left.\mathrm{CH}_{2}\right)_{8}, \quad d: 4.98(-\mathrm{OH}), d: 7.22(\mathrm{Ar}-\mathrm{H})_{2}$. The number of various $\mathrm{H}$ atoms obtained from HNMR spectrum analysis of the synthesis product accorded with the number of various $\mathrm{H}$ atoms in 2,6-di-tert-butyl-4-nonylphenol.

\subsection{Effect of Amount of the Catalyst}

These experiments have demonstrated an effect of the different amounts of catalyst (self-made composite super 
acid) on alkylation. Table 2 presents the data of influence of different catalyst amounts on the reaction time and the appearance of product at the temperature of $60-80{ }^{\circ} \mathrm{C}$

Table 2. Influence of the Catalyst Weight on Reaction Time and Appearance of Product

\begin{tabular}{|c||c|c|c|}
\hline Mass Fraction of Catalyst (\%) & 0.016 & 0.019 & 0.022 \\
\hline Time of Reaction (h) & 5 & 4 & 3 \\
\hline $\begin{array}{c}\text { Appearance and } \\
\text { Color of Product }\end{array}$ & qualified & qualified & non-qualified \\
\hline
\end{tabular}

According to the result from Table 2, it could be summarized that the time of reaction can be shorten as the amount of catalyst increases. If appearance of the product and the reaction rate were all taken into consideration, the best conditions of the alkylated reaction are to add $0.019 \%$ mass fraction of catalyst and react for approximately 4 hours.

\section{CONCLUSIONS}

(1) The catalyst of composite super acid was produced with the proportion of $m\left(\mathrm{C}_{5} \mathrm{~F}_{11} \mathrm{SO}_{3} \mathrm{H}\right): m\left(\mathrm{CF}_{3} \mathrm{SO}_{3} \mathrm{H}\right)$ : $m\left(\left(\mathrm{CF}_{3} \mathrm{SO}_{2}\right)_{2} \mathrm{O}\right)=0.8: 0.1: 0.1$. This catalyst has the advantage of high selectivity and catalytic activity. It has stronger acidity than inorganic acids and the ability to promote homogeneously catalyzed reaction. It can also reduce complex post-treatment procedure and the discharge of three wastes.

(2) The conditions of the synthesis of 2,6-di-tert-butyl-4nonylphenol are as follows: reaction temperature is $60-80^{\circ} \mathrm{C}$, reaction time is $4 \mathrm{~h}, n(4-\mathrm{n}$-nonyl phenol): $n$ (Isobutylene) $=1: 2$ and the amount of catalysts is $0.019 \%$ of the quantity of 4-n-nonyl phenol reactant, the yield of product can reach to $98 \%$.

(3) The result of elemental analysis is : found (calcd.) is $w(\mathrm{C})=83.07 \%(83.1 \%), \quad w(\mathrm{H})=11.85 \%(12.04 \%)$, $w(\mathrm{~N})=0(0) . \quad \operatorname{IR}\left(v / \mathrm{cm}^{-1}\right) \quad 3649.65,1156.67,767.97$, $1236.95,1438.84$. HNMR(CDC13), $d: 0.88\left(-\mathrm{CH}_{3}\right)_{7}$, $d: 1.58,1.43,1.23 .1 .13\left(-\mathrm{CH}_{2}\right)_{8}, \quad d: 4.98(-\mathrm{OH}), d: 7.22(\mathrm{Ar}-$
$\mathrm{H})_{2 .}$ The data of elemental analysis of the product, infrared spectrum and NMR accorded with the structure of 2,6-di-tert-butyl-4-nonylphenol.

\section{REFERENCES}

[1] Yang M. Plastic Additive Manual. Jiangsu Science Press: China, 2002, $80-86$

[2] Tim, C. Addictive trend. Lubricants World: USA, 1998, 11, 24-29.

[3] Sizhi, Z.; Wenfu, Q.; Pinliang, Z.; Fang, S.; Fuguang, Q. Development of liquid antioxidants for oils. Synth. Mater. Aging Appl., 2002, 3, 1-2.

[4] Kuilong, S.; Shaolin, C.; Jingyun, Y. Commercial application of a new liquid anti-oxidant T502A for gasoline. Petrol. Process. Petrochem., 2001, 1, 61-62.

[5] Hart, H.; Haglund, E. A. Orientation in the phosphoric acidcatalyzed alkylation of ortho-cresol. J. Org. Chem., 1956, 15, 396399.

[6] Kozhevnikov, I. V.; Tsyganok, A. I.; Timofeeva, M. N. Alkylation of methyl substituted phenols catalyzed by heteropoly acids. React. Kinet. Catal. Lett., 1992, 46(1), 17-23.

[7] Santacesaria, E.; Silvani, R.; Wilkinson, P.; Carra, S. Alkylation of p-cresol with isobutene catalyzed by cation-exchange resins: a kinetic study. Ind. Eng. Chem. Res., 1988, 27(4), 541-548.

[8] Yadav, G. D.; Pujari, A. A.; Joshi, A. V. Alkylation of p-cresol with methyl t-butyl ether (MTBE) over a novel solid acid catalyst UDCat-1. Green Chem., 1999, 1, 269-274

[9] Devassy, B. M.; Shanbhag, G. V.; Lefebvre, F.; Halligudi, S. B. Alkylation of p-cresol with tert-butanol catalyzed by heteropoly acid supported on zirconia catalyst. Mol. Catal. A, 2004, 210(1-2), 125-130.

[10] Chambers, R D. Fluorine in organic chemistry. John Wiley \& Sons: USA, 1973, 15-25.

[11] Olah, G. A.; Kaspi, J.; Bukala, J. Heterogeneous catalysis by solid super acids. 3. Alkylation of benzene and transalkylation of alkylbenzenes over raphite-intercalated Lewis acid halide and perfluorinated resin-sulfonic acid (Nafion-H) catalysts. Org. Chem., 1977, 42, 4187-4191.

[12] Gillespie, R.J.; Peel, T.E. The systems $\mathrm{H}_{2} \mathrm{SO}_{4}-\mathrm{HSO}_{3} \mathrm{~F} ; \mathrm{KSO}_{3} \mathrm{~F}-$ $\mathrm{HSO}_{3} \mathrm{~F} ; \mathrm{HSO}_{3} \mathrm{~F}-\mathrm{SO}_{3} ; \mathrm{HSO}_{3} \mathrm{~F}-\mathrm{AsFSl} \mathrm{HSO}_{3} \mathrm{~F}-\mathrm{SbFs}$; and $\mathrm{HSO}_{3} \mathrm{~F}_{-} \mathrm{SbF}_{5}-$ $\mathrm{SO}_{3}$. Am. Chem. Soc., 1973, 95, 5173-5178.

[13] Gillespie, R. J.; Peel, T. E. the Hammett acidity function for some super acid systems. I. The systems $\mathrm{H}_{2} \mathrm{SO}_{4}-\mathrm{SO}_{3}, \mathrm{H}_{2} \mathrm{SO}_{4}-\mathrm{HSO}_{3} \mathrm{~F}$, $\mathrm{H}_{2} \mathrm{SO}_{4}-\mathrm{HSO}_{3} \mathrm{Cl}$, and $\mathrm{H}_{2} \mathrm{SO}_{4}-\mathrm{HB}\left(\mathrm{HSO}_{4}\right)_{4}$. J. Am. Chem. Soc., 1971, 93, 5083-5087.

[14] Qingnian, D. Infrared spectroscopy. Chemical Industry Press: China, 1979, 128-129

[15] Haoqiang, Z. Industrial synthesis of B-carotene. Chem. Prod. Technol., 1997, 2, 14-17. 